\title{
Comparing Mindfulness-Based Group Therapy With Treatment as Usual for Opioid Dependents: A Pilot Randomized Clinical Trial Study Protocol
}

\author{
Saeed Imani ${ }^{1}$; Mohammad Kazem Atef Vahid ${ }^{2}$; Banafsheh Gharraee ${ }^{2}$; Mojtaba Habibi ${ }^{3}$; \\ Sarah Bowen ${ }^{4}$; Alireza Noroozi ${ }^{5,6, *}$ \\ ${ }^{1}$ Department of Clinical Psychology, Shahid Beheshti University, Tehran, IR Iran \\ ${ }^{2}$ Department of Clinical Psychology, Tehran Psychiatric Institute, Iran University of Medical Sciences, Tehran, IR Iran \\ 3 Family Research Institute, Shahid Beheshti University, Tehran, IR Iran \\ ${ }_{5}^{4}$ Addictive Behaviors Research Center, Department of Psychology, University of Washington, Seattle, USA

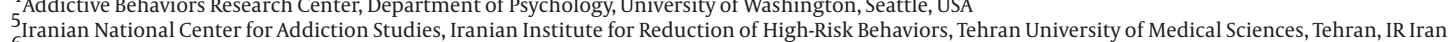 \\ 6 School of Advanced Technologies in Medicine, Tehran University of Medical Sciences, Tehran, IR Iran \\ ${ }^{*}$ Corresponding author: Alireza Noroozi, Iranian National Center for Addiction Studies, Tehran University of Medical Sciences, Tehran, IR Iran. Tel: +98-21-55421144, E-mail: \\ a_r_noroozi@yahoo.com
}

Received: January 11, 2014; Revised: October 24, 2014; Accepted: January 29, 2015

\begin{abstract}
Background: In response to high burden of opioid abuse in Iran, Ministry of Health has launched a large-scale opioid maintenance treatment program, delivered through a network of certified drug treatment centers. To promote opioid pharmacotherapies, there is an urgent need to develop and introduce evidence-based psychosocial interventions into the network.

Patients and Methods: This is a randomized clinical trial (RCT) to investigate feasibility and effectiveness of adding mindfulness-based group therapy to opioid pharmacotherapies as compared to opioid pharmacotherapies alone. The primary outcomes were treatment retention and percentage of weekly morphine, methamphetamine, and benzodiazepine negative tests.

Discussion: This is the first RCT that explores the effectiveness of mindfulness-based relapse prevention group therapy among opioid dependent clients in Iran. The feasibility of group therapy and comparison of outcomes in intervention and control groups should be discussed in the outcome article.
\end{abstract}

Keywords: Mindfulness; Pharmacotherapies; Therapy

\section{Introduction}

Iran has a long and hard-to-control border with Afghanistan, the main producer of illegal opiates in the world. Across this border opioid drugs are smuggled into the luxurious drug markets of European countries (1). According to a national epidemiologic study conducted by Ministry of Health, there are more than 1.2 million opioid dependents in the country (2). A more recent study estimated the number of drug dependents by indirect methods at 1.2 million that most of them were opioid users (3). A rapid change in opiate use patterns during recent years from opium to heroin and from smoking to injection has made the opioid dependence epidemic more problematic (4). Using unsterile syringe and injection equipment is the most prevalent route (69.2\%) of HIV transmission among all registered HIV positive cases (5). To respond to HIV epidemic among people who inject opioid, the methadone maintenance treatment was launched in 2002 and scaled up rapidly across the country.

Currently, opioid maintenance, treatment programs, and short-term medically assisted withdrawals are delivered through a network of certified drug treatment centers. Furthermore, opioid maintenance treatment could successfully decrease cue-induced cravings among opioid users (6), however, they are less effective in reducing stress-induced cravings (7). Hypothetically, new approaches of psychotherapies that focus on mindfulness and acceptance might enhance anti-craving properties of opioid maintenance treatment through reducing stressinduced cravings. Mindfulness-based therapies are new approaches in psychotherapy that integrate spiritual traditions such as mindfulness meditation with classic behavioral therapy approaches (8). Mindfulness helps clients to learn how to monitor their own mental processes free of judgment and without being caught up in the actual content of their thoughts. The core skill in mindfulness is the capacity to respond to aversive cognitions, sensations, and emotions with greater acceptance, present moment awareness, and a nonjudgmental attitude (9). Through mindfulness practices, the content of the thought becomes less important than the extent of the individual's awareness and reaction to it and its occurrence (9). 
Mindfulness-based therapies have proved to be effective in a wide range of physical and psychological problems such as chronic pain (10), depression, anxiety, and stress among college students (11) or people with subarachnoid hemorrhage (12), psychosocial stress (13), and depression (14), as well as distinct psychiatric disorders, including hypochondriasis (15), social phobia (16), generalized anxiety disorder (17), obsessive-compulsive disorders (18), and somatization disorder (19). Mindfulness-based relapse prevention (20) is a program integrating mindfulness meditation practices with traditional relapse prevention (RP). The program was tested and proved its effects in decreasing relapses in different groups of drug users, (21). Similar mindfulness-based programs for substance abuse have been shown to increase compliance, cognitive control, regulation of emotions, and reduce stress in alcohol dependents $(22,23)$ as well as some other similar influences (24).

The effectiveness of mindfulness in different populations of drug users suggests that more trials (in which the type of 'Mindfulness-based Group Therapy' was clearly defined and described) were required to establish the efficacy of Mindfulness-based Group Therapy for opioid dependence treatments.

\section{Patients and Methods}

This study was a pilot, randomized, parallel group, one to one controlled trial investigating feasibility, acceptability and effectiveness of mindfulness-based group therapy to improve treatment outcomes in opioid dependent clients.

\subsection{Inclusion Criteria}

The opioid dependent clients receiving treatment as usual in Iranian National Center for Addiction Studies (INCAS) were randomly assigned to intervention and control groups. INCAS Clinic is located in southwest of Tehran, Iran and provides assisted withdrawal and maintenance treatment with opioid agonist medications, including methadone and buprenorphine for its opioid dependent clients (96.6\% male). Inclusion criteria were as follows: (1) diagnosis of opioid dependence according to DSM-IV-TR criteria, (2) age range 18 to 40 years, (3) at least 8 years of completed education (5), stabilization on medical treatment (4), and informed written consent for participation in the study. The exclusion criteria were psychosis, dementia, imminent suicide risk, organic brain disorders, diagnosis of other drug dependence (except nicotine), or non-stabilization on 2-week medical treatments.

\subsection{Content of the Interventions}

Intervention was a combination of 8-session mindfulness-based group therapy developed by Bowen et al. and the usual treatment provided to opioid dependents in
INCAS clinic (Figure 1). The study was designed in a manner to be comparable to other studies of mindfulnessbased group therapy for addiction treatment. There was an English manual available to deliver the intervention (20), which described it as a structured intervention. One of the authors translated the manual to Farsi, which was edited by an addiction expert with a PhD in clinical psychology. The therapy sessions held in a closed-group format and conducted on a weekly basis. Each session lasted 120 minutes. It was a structured intervention with a predefined title and content for each session, and the sessions were kept in sequence. Table 1 outlines an overview of the topics and themes that were covered in each session. Although each session had a central theme, the sessions were intended to build upon previous week's practices.

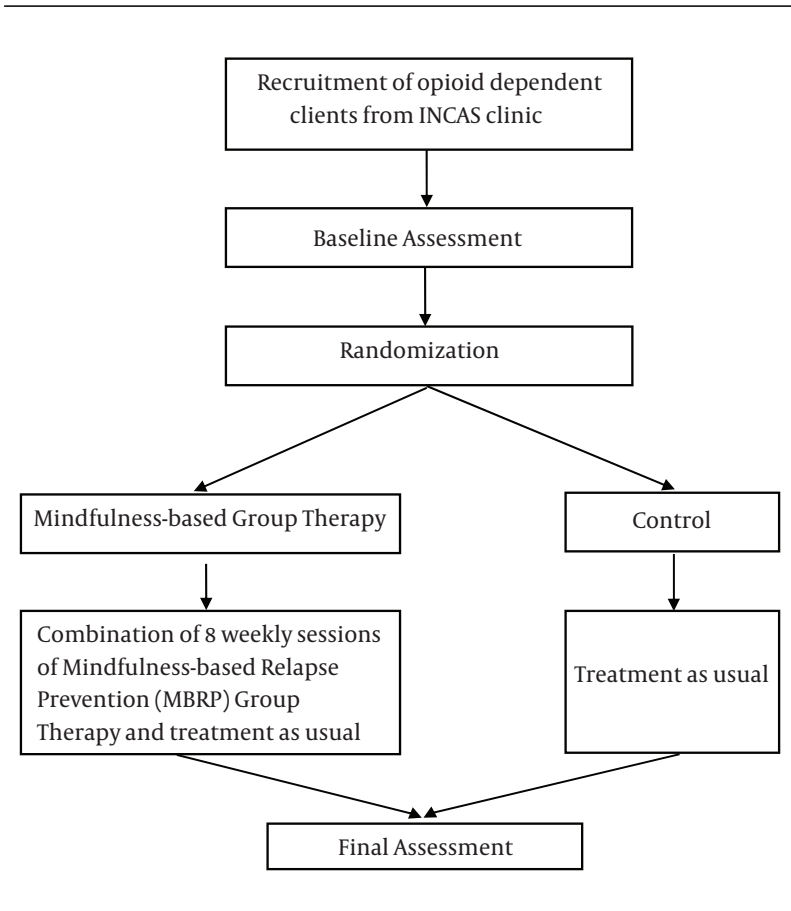

Figure 1. Consort Diagram of the Study

Table 1. Mindfulness-based Relapse Prevention Group Therapy Sessions

\begin{tabular}{lr}
\hline Session & Central Theme \\
\hline $\mathbf{1}$ & Automatic pilot and relapse \\
$\mathbf{2}$ & Awareness of triggers and cravings \\
$\mathbf{3}$ & Mindfulness in daily life \\
$\mathbf{4}$ & Mindfulness in high risk situations \\
$\mathbf{5}$ & Acceptance and skillful actions \\
$\mathbf{6}$ & Seeing thoughts as thoughts \\
$\mathbf{7}$ & Self-care and lifestyle balance \\
$\mathbf{8}$ & Social support and continuing practice \\
\hline
\end{tabular}


Imani S et al.

Table 2. Assessment Instruments Proposed for the Current Study

\begin{tabular}{|c|c|c|c|}
\hline Instrument & Baseline & Weekly & 8 Weeks Follow up \\
\hline Log sheet of treatment participation & & $\sqrt{ }$ & \\
\hline Urine drug testing for morphine, methamphetamine, and benzodiazepines & $\sqrt{ }$ & $\sqrt{ }$ & $\sqrt{ }$ \\
\hline Addiction Severity Index (26) & $\sqrt{ }$ & & $\sqrt{ }$ \\
\hline Alcohol and drug cravings (27) & $\sqrt{ }$ & & $\sqrt{ }$ \\
\hline DASS-21 (19) & $\sqrt{ }$ & & $\sqrt{ }$ \\
\hline Mindfulness (28) & $\sqrt{ }$ & & $\sqrt{ }$ \\
\hline Acceptance (8) & $\sqrt{ }$ & & $\sqrt{ }$ \\
\hline
\end{tabular}

All study participants received medical treatment through opioid agonist medications in outpatient INCAS clinic according to national protocols (25). The methadone or buprenorphine administration was conducted through regular visits by certified physicians that were more frequent during the first week of treatment and gradually decreased to at least one visit per week. Clients who received maintenance treatment should have come to the clinics for daily supervised dosing during the first two months of treatment. The take-home doses were allowed $1 \mathrm{~d} / \mathrm{wk}$ from the beginning of the third month, which could increase from $1 \mathrm{~d} /$ mon to at most $6 \mathrm{~d} / \mathrm{wk}$ contingent to appropriate compliance and negative weekly urine tests for morphine and methamphetamine. The baseline, weekly, and end of the treatment study assessments are presented in Table 2.

\subsection{Sample Size Calculation}

The study participants recruited in INCAS in the autumn of 2013. Thirty eligible individuals were selected randomly for participation in the study; 15 of them were assigned to each intervention group. In order to have consistency in seasonality across assessment points, the assessment obtained in the autumn of 2013 served as the baseline or pretest assessment point for the main substantive intervention outcome analysis and the winter of 2013 assessment served as the outcome time point for the analysis report; hence, this was an experimental research design.

\subsection{Statistical Analysis}

To determine legitimate numerical codes, data cleaning was performed by both graphical and descriptive statistics to ensure data accuracy, check out-of-range values for categorical variables, and make sure that mean and standard deviations were within the plausible ranges for continuous composite variables (29). The patterns of missing data were checked regarding how much and why they were missing. Results of SPSS MVA (missing values analysis) using t test $(\alpha=0.05)$ for outcome composite variables were checked to ensure that patterns of missing data in outcome variables were not related to population's demographic variables such as sex, area of residency, and group (intervention vs. condition). Distribution of scores on continuous variables was checked for univariate $(\mathrm{P}<0.001)$ and multivariate normality using LISREL, version 8.72 (30). T test and chi-square test were conducted to match the intervention and control groups with respect to demographic variables. Repeated measure analysis of variance was used to test the effectiveness of mindfulness-based group therapy in the study population.

\section{Discussion}

To the best of our knowledge, this is the first study to test the acceptability and efficacy of 'Mindfulness-based Group Therapy' versus ‘Treatment as Usual' for opioid dependent clients in Iran. There are some potential limitations regarding the present study that merit discussion. One potential concern is the random assign of the participants to their study condition, which may lead to realize whether or not they are receiving the added group therapy. When the participants prefer one group to the other, this might disappoint them, which may negatively affect the treatment outcome. However, since the study intended to investigate the effect of adding the mindfulness-based group therapy to treatment as usual, the current design remains most appropriate as it is closer to a real world situation. After the mindfulness-based group therapy session, the study participants were advised to report their participation in self-help programs during the course of the therapy. As it is not possible to monitor participants' behavior during this period, self-reports about their use of other treatment programs should be trusted. Throughout the recruitment, the mindfulnessbased group therapy sessions and its follow-ups, it was emphasized to the participants that their honest information was crucial and there would be no consequences if they participated in other methods. It is hoped that by conveying this message to the participants, they were motivated to cooperate throughout the study and provide their honest reports about their experiences during study assessments. The results of the present study indicate whether mindfulness-based group therapy can improve the outcome of opioid dependence treatment in the context of Iran drug treatment services.

\section{Acknowledgements}

Authors acknowledge the close collaboration of Iranian 
National Center for Addiction Studies (INCAS) director and staff for their kind assistance.

\section{Authors' Contributions}

Saeed Imani prepared the first draft of the paper and coordinated the study. Mohammad Kazem Atef Vahid was the principal investigator, developed the study design and prepared the final draft of the paper. Alireza Noroozi, Banafsheh Gharraee, Mojtaba Habibi, and Sarah Bowen critically reviewed the study design and developed the analysis methods. All authors read and approved the final version of the manuscript.

\section{Funding/Support}

The first author received a small grant from Tehran University of Medical Sciences (TUMS) to conduct the study as his $\mathrm{PhD}$ thesis. Other authors have not received any financial support for the study.

\section{Declaration of interest}

None Declared.

\section{References}

1. United Nation Office of Drug and Crime. World Drug Report .Vienna: UNODC Publications; 2013.

2. Yasami MT, Shahmohammadi D, Naghavi M, Bagheri-Yazdi A, Zojaji A, Rahimi-Movaghar A. Epidemiology of drug abuse in I.R. Iran. Ministry of Health and Drug Control Headquarters. 2002.

3. Narenjiha H, Rafiey H, Jahani MR, Assari S, Moharamzad Y, Roshanpazooh M. Substance-dependent professional drivers in Iran: a descriptive study. Traffic Inj Prev. 2009;10(3):227-30.

4. Malekinejad M, Vazirian M. Transition to injection amongst opioid users in Iran: implications for harm reduction. Int J Drug Policy. 2012;23(4):333-7.

5. Ministry of Health and Medical Education. [Quarterly reported of statistics of HIV/AIDS Cases Ministry of Health and Medical Education, Center of Diseases Management, Sexually Transmitted Disease and AIDS Office].; 2012.

6. Shi J, Zhao LY, Epstein DH, Zhang XL, Lu L. Long-term methadone maintenance reduces protracted symptoms of heroin abstinence and cue-induced craving in Chinese heroin abusers. Pharmacol Biochem Behav. 2007;87(1):141-5

7. Ilgen M, Jain A, Kim HM, Trafton JA. The effect of stress on craving for methadone depends on the timing of last methadone dose. Behav Res Ther 2008;46(10):1170-5.

8. Strosahl KD, Wilson KG, Bissett RT, Pistorello J, Toarmino D. Measuring experiential avoidance: A preliminary test of a working model. Psychol Rec. 2004;54(4):553-78.

9. Segal Z, Williams JMG, Teasdale J. Mindfulness-based cognitive therapy for depression.New York: Guilford Press; 2002.

10. Leigh J, Bowen S, Marlatt GA. Spirituality, mindfulness and substance abuse. Addict Behav. 2005;30(7):1335-41.

11. Masuda A, Tully EC. The role of mindfulness and psychological flexibility in somatization, depression, anxiety, and general psy- chological distress in a nonclinical college sample. J Evid Based Complementary Altern Med. 2012;17(1):66-71.

12. Joo HM, Lee SJ, Chung YG, Shin IY. Effects of mindfulness based stress reduction program on depression, anxiety and stress in patients with aneurysmal subarachnoid hemorrhage. J Korean Neurosurg Soc. 2010;47(5):345-51.

13. Morone NE, Lynch CP, Losasso III VJ, Liebe K, Greco CM. Mindfulness to reduce psychosocial stress. Mindfulness. 2012;3(1):22-9.

14. Song Y. Depression, Stress, Anxiety and Mindfulness in Nursing Students. Korean J Adult Nurs. 2011;23(4):397-402.

15. McManus F, Surawy C, Muse K, Vazquez-Montes M, Williams JM. A randomized clinical trial of mindfulness-based cognitive therapy versus unrestricted services for health anxiety (hypochondriasis).J Consult Clin Psychol. 2012;80(5):817-28.

16. Schmertz SK, Masuda A, Anderson PL. Cognitive processes mediate the relation between mindfulness and social anxiety within a clinical sample. J Clin Psychol. 2012;68(3):362-71.

17. Evans S, Ferrando S, Findler M, Stowell C, Smart C, Haglin D. Mindfulness-based cognitive therapy for generalized anxiety disorder.J Anxiety Disord. 2008;22(4):716-21.

18. Hertenstein E, Rose N, Voderholzer U, Heidenreich T, Nissen C, Thiel N, Herbst N, Kulz AK, et al. Mindfulness-based cognitive therapy in obsessive-compulsive disorder - a qualitative study on patients' experiences. BMC Psychiatry. 2012;12:185.

19. Fjorback LO, Arendt M, Ornbol E, Walach H, Rehfeld E, Schröder A Fink P, et al. Mindfulness therapy for somatization disorder and functional somatic syndromes-Randomized trial with one-year follow-up. J Psychosom Res. 2013;74(1):31-40.

20. Bowen S, Chawala N, Marlatt GA. Mindfulness-Based Relapse Pervention for Addictive Behaviors.New York: Guilford Press; 2011

21. Bowen S, Chawla N, Collins SE, Witkiewitz K, Hsu S, Grow J, Clifasefi S, Garner M, Douglass A, Larimer ME, Marlatt A, et al. Mindfulness-based relapse prevention for substance use disorders: a pilot efficacy trial. Subst Abus. 2009;30(4):295-305.

22. Garland EL. Trait Mindfulness Predicts Attentional and Autonomic Regulation of Alcohol Cue-Reactivity. J Psychophysiol. 2011;25(4):180-9.

23. Garland EL, Schwarz NM, Kelly A, Whitt A, Howard MO. Mindfulness-Oriented Recovery Enhancement for Alcohol Dependence: Therapeutic Mechanisms and Intervention Acceptability. J Soc Work Pract Addict. 2012;12(3):242-63.

24. Chiesa A, Serretti A. Are mindfulness-based interventions effective for substance use disorders? A systematic review of the evidence. Subst Use Misuse. 2014;49(5):492-512.

25. Ministry of Health and Medical Education. National Protocol for Treatment of Opioid Dependence with Opioid Agonist Medications Substance Abuse Prevention and Treatment Office (SAPTO), Bureau of Psychosocial Health and Addiction.Tehran: Ministry of Health and Medical Education; 2006.

26. McLellan AT, Kushner H, Metzger D, Peters R, Smith I, Grissom G Pettinati $\mathrm{H}$, Argeriou M, et al. The Fifth Edition of the Addiction Severity Index. J Subst Abuse Treat. 1992;9(3):199-213.

27. Flannery BA, Volpicelli JR, Pettinati HM. Psychometric properties of the Penn Alcohol Craving Scale. Alcohol Clin Exp Res. 1999;23(8):1289-95.

28. Baer RA, Smith GT, Hopkins J, Krietemeyer J, Toney L. Using selfreport assessment methods to explore facets of mindfulness. Assessment. 2006;13(1):27-45.

29. Tabachnick BG, Fidell LS. Using multivariate statistics. 5th edNew York: HarperCollins; 2007.

30. Joreskog KG, Sorbom D. LISREL 8: User's Reference Guide.Chicago: Scientific Software, Inc.; 2003. 